\title{
Phase camera development for gravitational wave detectors
}

\author{
Kazuhiro Agatsuma* \\ National Institute for Subatomic physics, Amsterdam \\ E-mail: agatsuma@nikhef.nl \\ David Rabeling \\ The Australian National University, Canberra \\ E-mail: david.rabelingeanu.edu.au

\section{Martin van Beuzekom} \\ National Institute for Subatomic physics, Amsterdam \\ E-mail: martinbenikhef.nl

\section{Jo van den Brand} \\ National Institute for Subatomic physics, Amsterdam \\ VU University, Amsterdam \\ E-mail: jo@nikhef.nl
}

\begin{abstract}
A preliminary experiment and usage of the phase camera is reported on here. The phase camera is a frequency selective wavefront sensor of the laser beam, which is utilized for observing phasemodulated laser beam (sidebands) in an interferometric gravitational wave detectors. The laser modulation/demodulation technique plays an essential role in readout of the mirror displacement for accurate position controls. The sideband signals in the power recycling cavity are easily degraded by mirror aberrations in the configuration of a marginally stable cavity. Recently, the phase camera is being applied for monitoring such aberrations in Advanced Virgo. The observed aberrations will be corrected by $\mathrm{CO}_{2}$ lasers and compensation plates.
\end{abstract}

Technology and Instrumentation in Particle Physics 2014,

2-6 June, 2014

Amsterdam, the Netherlands

\footnotetext{
* Speaker.
} 


\section{Background}

The gravitational wave (GW) detectors are now well sophisticated interferometer (IFO) as a displacement sensor to measure the spacetime deformation by GWs. To operate IFO, it is necessary to control the position and alignment of mirrors. The Pound-Drever-Hall technique is used to obtain displacement signals of the mirrors for their control [1]. In this technique, a condition of the sidebands created by phase modulation is related to the quality of the control directly. The laser sideband signals become significant not only for the controls but also for the sensitivity of detectors because the control loops can introduce noise.

The GWs are expected to be detected by the next generation GW detectors. Advanced Virgo, which is an upgrade of Virgo, is one of them [2]. Advanced Virgo will employ a marginally stable configuration in the power recycling cavity (PRC). It means that the higher order modes of cavity are easily exited because the gouy phase becomes close to zero. The main origin of the higher order modes are aberrations of mirrors, like thermal lensing, substrate inhomogeneities, and surface shape errors. By such higher order modes, the power recycling gain and included sideband signals are decreased i.e. controls become unstable or even impossible.

In order to suppress aberrations, a thermal compensation system (TCS) will be utilized [3]. The Hartmann sensor [4] and phase camera will be used in the TCS as the wavefront sensor, which can make aberration maps, but the Hartmann sensor can detect only thermal aberrations. Instead, the phase camera becomes useful tool to observe whole aberrations including optical aberrations in the PRC. According to simulations [5], keeping the PRC at resonance becomes challenging for aberrations of $5 \mathrm{~nm}$ RMS or larger. The phase camera allows to observe the aberrations of $1 \mathrm{~nm}$ level or better. After obtaining the aberration information, it can be corrected using $\mathrm{CO}_{2}$ lasers and compensation plates [6]. Thus, the phase camera is being developed for observing aberrations in a $\mathrm{GW}$ detector. It is useful not only for the aberration scanning, but also for monitoring the condition of sidebands in general.

\section{Principle}

The phase camera is constructed by a combination of the heterodyne detection and pinhole scanning [7]. As shown in figure 1 the heterodyne beam (reference beam) is created by picking off the main beam of IFO and by shifting frequency using an acousto-optic modulator (AOM). The test beam, which includes sideband signals made by electro-optic modulator (EOM), is picked off from an observation point in IFO. After combining the reference beam and test beam at a beam splitter, the combined laser signal is detected by a photo detector (PD) and demodulated by the beat frequencies between the heterodyne frequency and phase modulation frequencies. The amplitude and phase signals are constructed from two different demodulation phases (the so-called $I$ phase and $Q$ phase). In addition to this heterodyne detection, a scanner can change a fraction of the wavefront projected onto the pinhole PD. By scanning the wavefront, mapping patterns of amplitude and phase are obtained. The mapping patterns show the state of sidebands. All sidebands including upper and lower sidebands become selective by preparing a suitable heterodyne frequency $f_{\mathrm{H}}$, which can make each different modulation frequency $\left(f_{\mathrm{H}}, f_{\mathrm{H}} \pm f_{\mathrm{p} 1}, f_{\mathrm{H}} \pm f_{\mathrm{p} 2}, \cdots\right)$. 


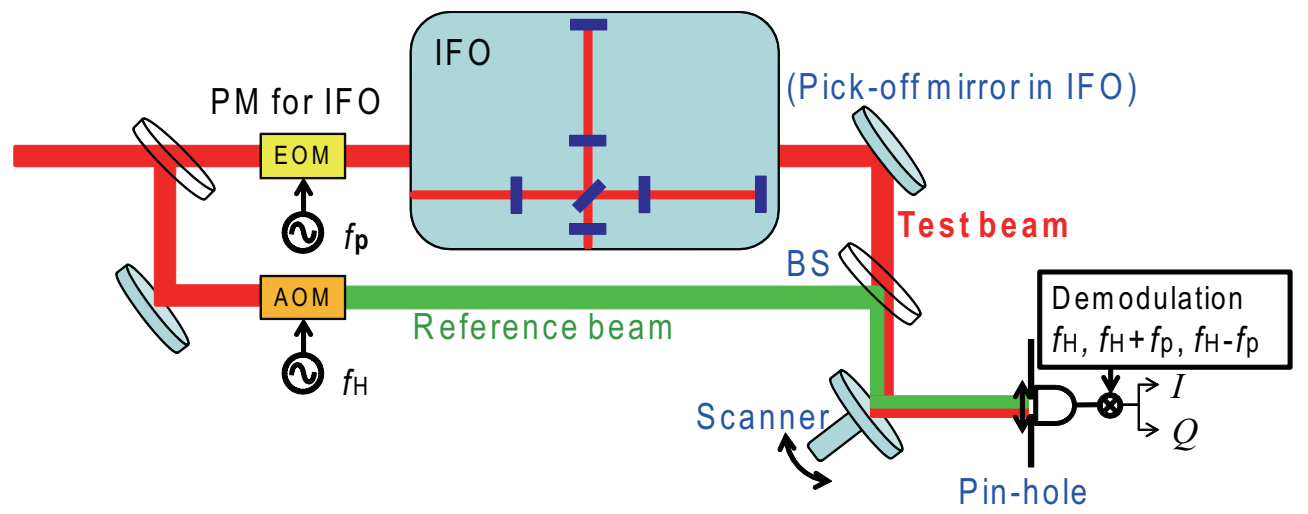

Figure 1: Principle of the phase camera. EOM, electro-optic modulator; AOM, acousto-optic modulator; PM, phase modulation; IFO, interferometer; BS, beam splitter; $f_{\mathrm{p}}$, phase modulation frequency; $f_{\mathrm{H}}$, heterodyne frequency.

\section{Use of phase camera in Advanced Virgo}

For operating the IFO, five different frequencies are used for phase modulation in Advanced Virgo. Three detection ports of the phase camera will be placed on the external injection bench, small external table next to the PR-pick-off minitower, and external detection bench, to observe input beam, PRC, and output beam, respectively (see figure 2). In order to share the identical reference beam for every detection port, a fiber coupled AOM is set on PSL bench.

All of the sidebands can be monitored at PC1. It is useful to check the condition of sidebands before entering IFO. The sidebands of $\mathrm{f} 1, \mathrm{f} 2$, and $\mathrm{f} 4$ can be observed at PC2. The sideband of $\mathrm{f} 1$ is used for controlling the common mode of the PRC. The sideband of $\mathrm{f} 4$ is prepared to support $\mathrm{f} 1 \mathrm{in}$ high aberration condition because $\mathrm{f} 4$ is insensitive to aberrations due to a low finesse in the cavity. The aberration map is obtained from a subtraction between phase patterns of a sideband of $\mathrm{f} 1$ and it of the carrier because these two patterns should be identical in an ideal IFO (no aberration). The information of aberration map from PC2 is fed back to $\mathrm{CO}_{2}$ laser. The $\mathrm{CO}_{2}$ laser will make a correction pattern of the aberration onto a compensation plate placed on near side of the front mirror. The $\mathrm{f} 2$ sideband, which is used for controlling the signal recycling cavity, is monitored at PC3. Also, the aberration map of the PRC differential mode can be obtained at PC3.

\section{Prototype test}

\subsection{Experimental setup}

We are testing the prototype of the phase camera at Nikhef before installation on the Virgo site [10]. A photograph is shown in figure 3. The laser source is a Nd:YAG laser with a wavelength of $1064 \mathrm{~nm}$ and with a nominal power of $500 \mathrm{~mW}$. The test beam is made by a broadband EOM (DC to $250 \mathrm{MHz}$ ) without interferometer for simplicity. The reference beam is created by a fiber coupled AOM with a frequency shift of $80 \mathrm{MHz}$. By selecting the heterodyne frequency of $80 \mathrm{MHz}$, each modulation frequency has individual frequency, i.e. both the upper and lower sidebands (10 sidebands in total) can be probed. A galvanometer (GVS012, Thorlabs inc.) is used as a scanner. 


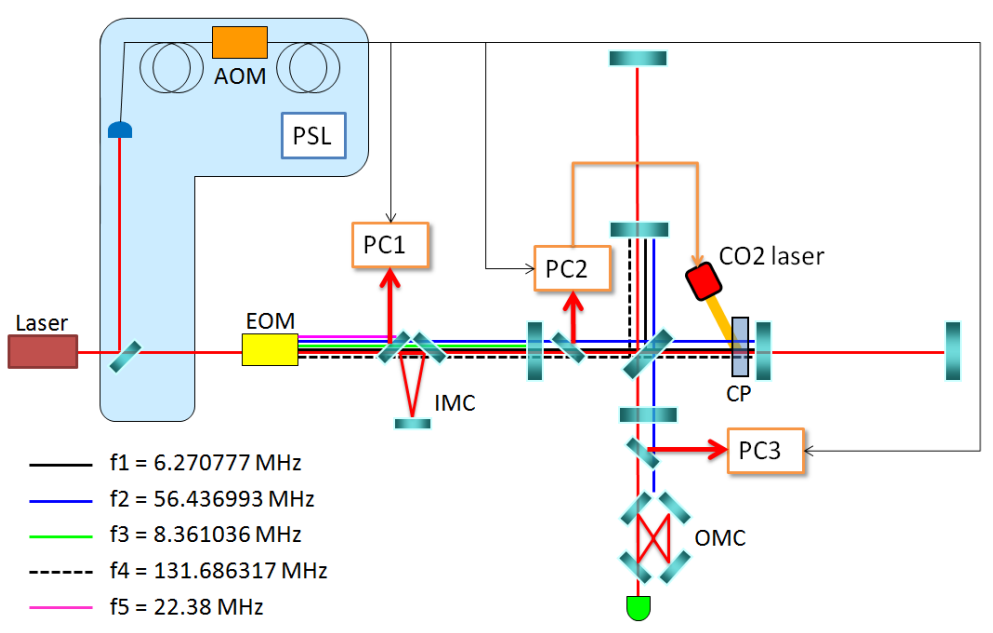

Figure 2: Installation of the phase camera for Advanced Virgo. Three detection ports of the phase camera will be installed (PC1, PC2, and PC3). Five lines show different phase modulations (f1 - f5). Each of them makes both upper and lower sidebands around carrier frequency. PSL, pre-stabilized laser bench; PC, phase camera; $\mathrm{CP}$, compensation plate; IMC, input mode cleaner; OMC, output mode cleaner.

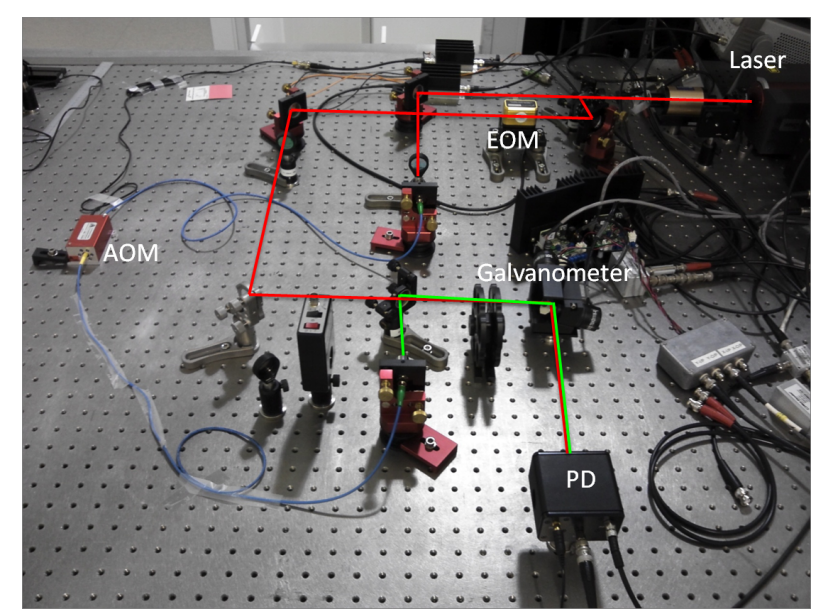

Figure 3: Prototype experiment at Nikhef. The red line shows the input beam and test beam with a phase modulation by EOM. The green line shows the reference beam whose frequency is shifted by AOM.

The current PD is New focus 1811 (125 MHz), which has an aperture of $300 \mu \mathrm{m}$. Demodulation is performed by ADC/FPGA board (400 MHz clock).

\subsection{Preliminary result}

A preliminary result is shown in figure 4 The phase modulation frequency was $10 \mathrm{MHz}$ and the modulation index was about $0.06 \mathrm{rad}$. The coaxial interference pattern in figure 4 (c) is a spatial fringe pattern between TEM00 modes of the test beam and reference beam. The edge side of figure 4(d) suffers from low signal to noise ratio. From the subtraction data of figure 4 (b) and (d), an aberration mapping can be obtained. 

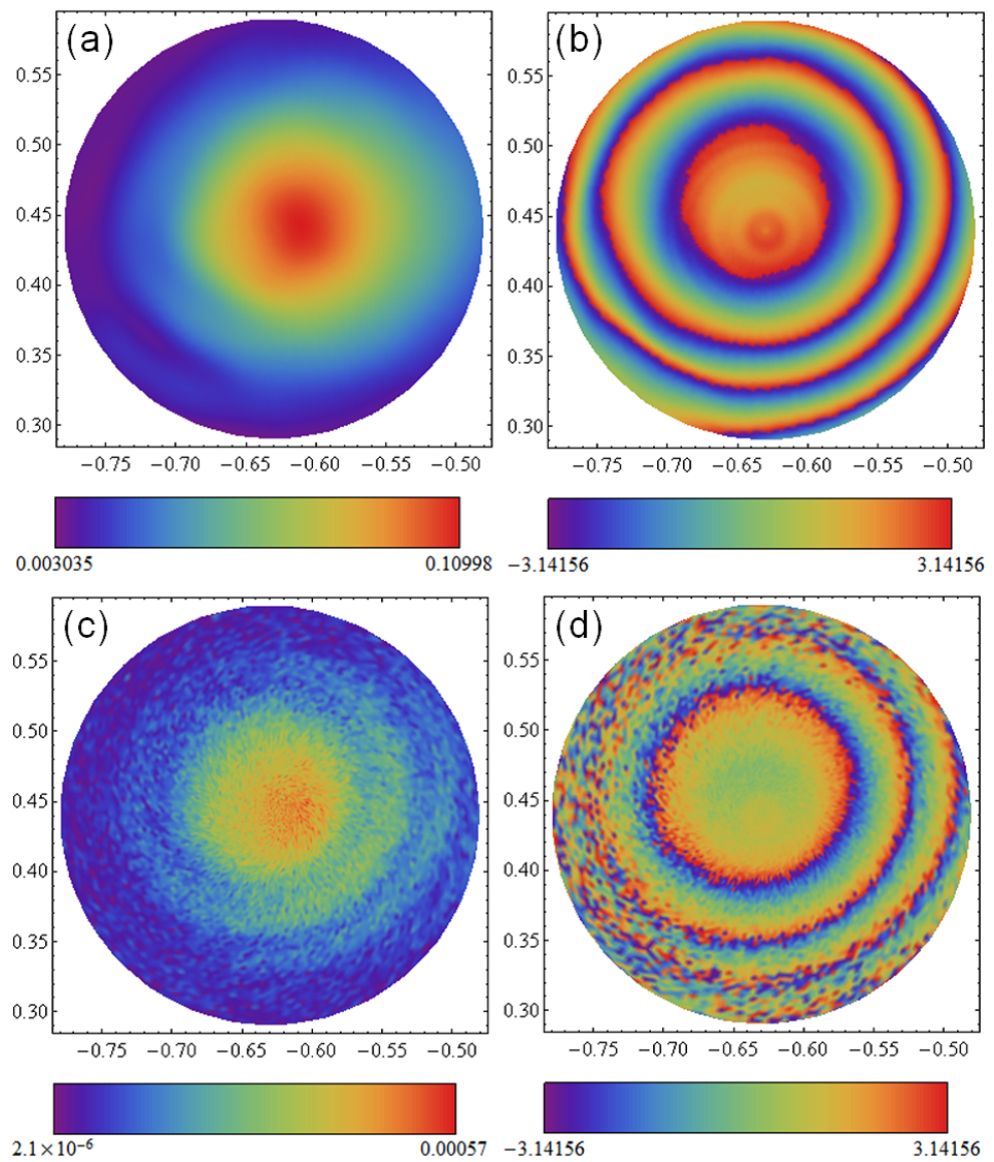

Figure 4: Preliminary result of the mapping. The vertical and horizontal axises indicate transversal two directions of wavefronts (arbitrary units). (a); amplitude of carrier, (b); phase of carrier, (c); amplitude of upper sideband, (d); phase of upper sideband.

\subsection{Scanning pattern}

The Archimedes' spiral is planned to use as the scanning pattern. A formula of the Archimedes' spiral is written as $r=a \theta$, where $r$ is radius of the spiral, $a$ amplitude of spiral (time dependent), $\theta$ rotation angle. In the Cartesian coordinates, it is rewritten as $X=\left(A t / 2 T_{\mathrm{s}}\right) \cos \left(2 \pi f_{\mathrm{s}} t\right)$ and $Y=\left(A t / 2 T_{\mathrm{s}}\right) \sin \left(2 \pi f_{\mathrm{s}} t\right)$. Here, $A$ is the diameter of scanning area, $t$ time, $T_{\mathrm{s}}$ the total acquisition time, and $f_{\mathrm{s}}$ the rotation frequency of the spiral. The rotation frequency $f_{\mathrm{s}}$ is given by

$$
f_{\mathrm{s}}=\frac{\sqrt{N_{\mathrm{p}}}}{2 T_{\mathrm{s}}}
$$

to make an uniform spiral pattern $\left(\sqrt{N_{\mathrm{p}}} / 2\right.$ times rotation during $T_{\mathrm{s}} ; N_{\mathrm{p}}$ is the pixel number). It contains three arbitrary parameters, $A, T_{\mathrm{s}}$, and $N_{\mathrm{p}}$. However, these values are limited by requirements and the smallest difference among the demodulation frequencies.

The scanning area is fixed to a diameter of $5 \mathrm{~mm}$, which comes from a typical aperture size on the optical benches in Virgo. The required minimum density is $100 \times 100$ pixels [8] (actually, $128 \times 128$ pixels are used due to a binary compatibility). Even if no limitation of a mechanical 
scanning speed, a total acquisition time of at least $2^{-5} \mathrm{~s}(0.03 \mathrm{~s})$ is necessary to distinguish two different demodulation frequencies. The smallest difference is $\Delta f=\mathrm{f} 3-\mathrm{f} 1=2.09 \mathrm{MHz}$. A four times smaller frequency bin (about $0.5 \mathrm{MHz}$ ) is, at least, necessary to resolve these peaks in the frequency spectrum. The frequency resolution $f_{\text {res }}$ is related to the acquisition time by $T_{\mathrm{s}}=N_{\mathrm{p}} / f_{\text {res }}$. Thus, the minimum acquisition time is naively estimated from $N_{\mathrm{p}} / \Delta f=2^{14} /\left(0.5 \times 10^{6}\right)=2^{-5} \mathrm{~s}$. A simulation result [9] also accord with this estimation. Although a quicker feedback is better for TCS typically, the scanning speed of $1 \mathrm{~Hz}$ or more (i.e. total acquisition time of $1 \mathrm{~s}$ or less) is enough because the thermal effects (aberrations) are expected to be below about $0.1 \mathrm{~Hz}$.

From the above discussion, there are valid ranges of the scanning parameters $\left(N_{\mathrm{p}} \geq 128 \times 128\right.$, $0.03 \leq T_{\mathrm{s}} \leq 1$, corresponding to $64 \leq f_{\mathrm{s}} \leq 2000$ ). Figure 5 shows examples of the scanning pattern by changing a parameter $N_{\mathrm{p}}\left(f_{\mathrm{s}}\right.$ is also changed by the relation of equation 4.1). A sufficient density is necessary more than (b). In practical terms, the quickest operation is not $0.03 \mathrm{~s}$ but is limited by a performance of the scanner.

(a)

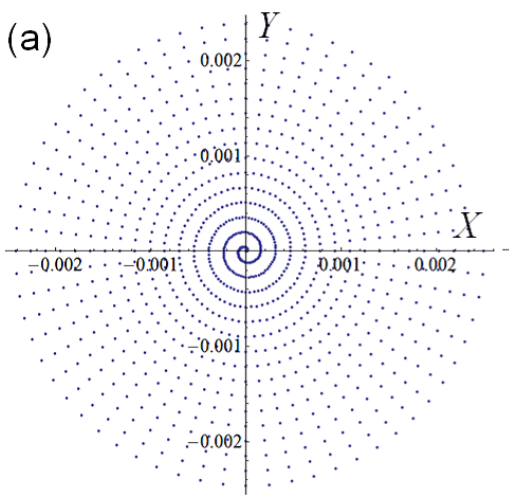

(b)

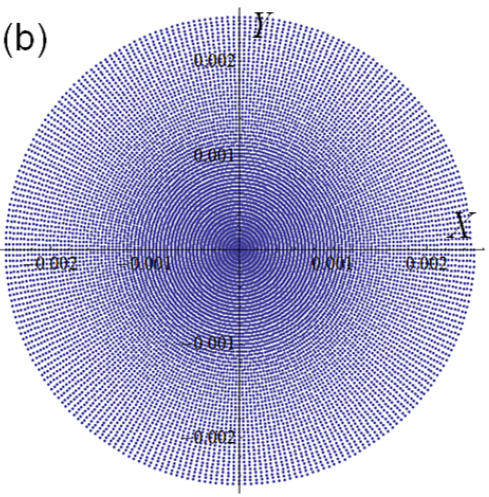

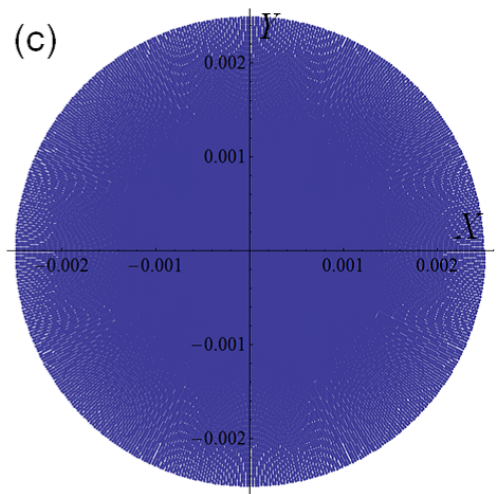

Figure 5: Examples of scanning pattern (Archimedes' spiral). The total acquisition time is fixed at $1 \mathrm{~s}$. (a) $32 \times 32$ pixels, $16 \mathrm{~Hz}$ (the rotation frequency of a scanner). (b) $128 \times 128$ pixels, $64 \mathrm{~Hz}$. (c) $256 \times 256$ pixels, $128 \mathrm{~Hz}$.

\section{Components}

Components of the prototype are being upgraded after the initial tests as described in the previous section. The galvanometer produces sound while in operation, which makes it not suitable for table top experiments. The current PD response $(125 \mathrm{MHz})$ is still too slow to observe every sideband (the highest modulation frequency is $212 \mathrm{MHz}$ ). By considering Nyquist frequency, the suitable sampling frequency is higher than $424 \mathrm{MHz}$. The improved and selected components are shown in this section.

\subsection{Scanner}

According to the discussion of section 4.3 , a broad range (sweeping range of $5 \mathrm{~mm}$ ) and quick response (more than $64 \mathrm{~Hz}$ up to $2000 \mathrm{~Hz}$ ) is requested for the scanner. A PZT scanner (S-334, Physik Instrumente (PI) $\mathrm{GmbH} \& \mathrm{Co}$.) is selected as a suitable scanner, which has a range of 
$\pm 25 \mathrm{mrad}$ and high frequency response without sound noise. The necessary sweeping range depends on the distance from the scanner to the pinhole PD. The expected available distance is $20 \mathrm{~cm}$ because of the compatibility with other optics. To cover $5 \mathrm{~mm}( \pm 2.5 \mathrm{~mm})$ with the distance of $20 \mathrm{~cm}$, an angular range of $\pm 12.5 \mathrm{mrad}$ is needed. This is a half of the maximum movable range. It means this scanner can be operated up to a half of the maximum voltage. Figure 6 shows the frequency response of the scanner. The output voltage is reduced by an increase of the frequency. The half of the maximum point is about $300 \mathrm{~Hz}$. Thus, the operation frequency is limited in $64 \leq f_{\mathrm{s}} \leq 300$. The quickest operation becomes $0.25 \mathrm{~s}$ in this system to obtain one scanning pattern $(128 \times 128$ pixels, $256 \mathrm{~Hz})$. Incidentally, hysteresis of the PZT is suppressed by feedback controls with strain gauge sensor inside. The resonance of PZT is about $1 \mathrm{kHz}$, which is higher than operation frequency.

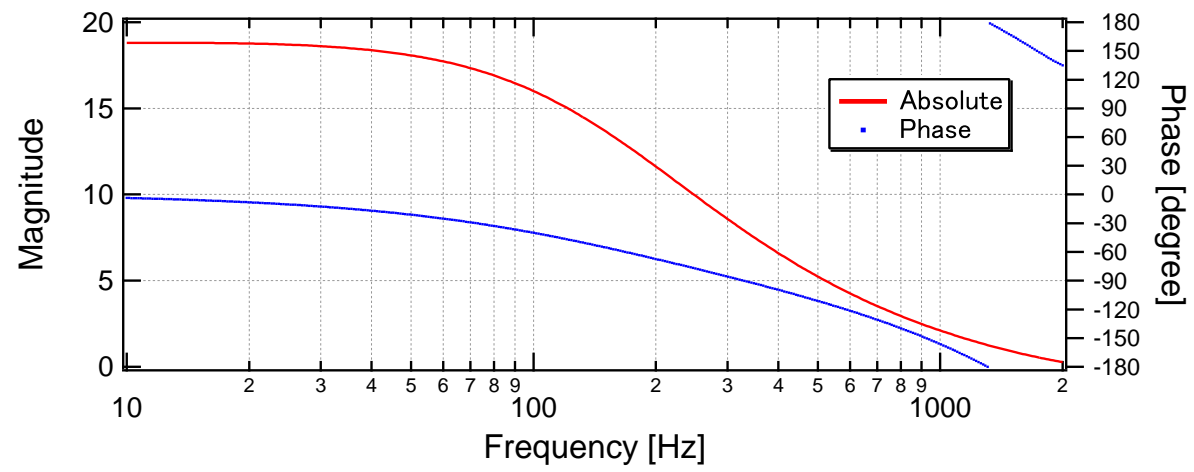

Figure 6: Frequency response of the scanner.

\subsection{Photo detector}

A new PD is being developed. A high speed InGaAs PD (FCI-InGaAs-55, OSI optoelectronics) is selected as the element of the PD. The active area diameter is $55 \mu \mathrm{m}$ that becomes the pinhole detection. The optical noise equivalent power is $2.66 \times 10^{-15} \mathrm{~W} / \sqrt{\mathrm{Hz}}$. The frequency response is almost flat up to $700 \mathrm{MHz}$, limited by an amplifier. The RF transimpedance amplifier is HMC799LP3E (HITTITE), which has $10 \mathrm{kOhm}$ transimpedance over $700 \mathrm{MHz}$ bandwidth and low noise $(46 \mathrm{nV} / \sqrt{\mathrm{Hz}}$ output noise, corresponding to $4.6 \mathrm{pA} / \sqrt{\mathrm{Hz}}$ input referred noise at around $100 \mathrm{MHz}$ ). By comparing the input noise level to the shot noise of the photodiode ( $\sqrt{2 e I_{\mathrm{p}}} ; e$ is the elementary charge and $I_{\mathrm{p}}$ is the diode current), the shot noise dominant performance is obtained when the diode current is more than $66 \mathrm{uA}$.

\subsection{Digital board}

By using digital processing unit, it becomes easy to demodulate 11 different frequencies (the heterodyne frequency and the sidebands of $\mathrm{f1}$ - f5) at the same time. This digital board is also being developed and assembled with 14 bit ADC at 500 MS/s and Virtex-7 FPGA (Xilinx). This can measure $32 \mathrm{k}$ pixels per second and have a frequency resolution of about $30 \mathrm{kHz}$. A measured standard deviation is $0.3 \mathrm{mrad}$ at $212 \mathrm{MHz}$ (the highest modulation frequency; the lower sideband of f4). 


\section{Summary}

The phase camera can observe the laser wave fronts with a frequency selective property. This is a useful monitor for sidebands, which is significant for the control of mirrors in GW detectors. An application of this system is in progress for monitoring aberrations, especially in Advanced Virgo. Prototype experiment is on going at Nikhef. Components have been selected and are being tested. The quickest scanning is $0.25 \mathrm{~s}$ with the selected PZT scanner (by $20 \mathrm{~cm}$ distance). It is sufficient for TCS. A high speed PD and digital board are being prepared.

\section{Acknowledgements}

We thank Guido Visser, Hans Verkooijen, and Mesfin Gebyehu for their technical supports. This work is part of the research programme of the Foundation for Fundamental Research on Matter (FOM), which is part of the Netherlands Organisation for Scientific Research (NWO).

\section{References}

[1] R. W. P. Drever, J. L. Hall, F. V. Kowalski, J. Hough, G. M. Ford, a. J. Munley, and H. Ward, Laser phase and frequency stabilization using an optical resonator, Appl. Phys. B Photophysics Laser Chem., vol. 31, no. 2, pp. 97-105, Jun. 1983.

[2] Virgo collaboration, Advanced Virgo Technical Design Report, Virgo Technical Documentation System (VIR-0128A-12)

[3] A. Rocchi, E. Coccia, V. Fafone, V. Malvezzi, Y. Minenkov, and L. Sperandio, Thermal effects and their compensation in Advanced Virgo, J. Phys. Conf. Ser., vol. 363, p. 012016, Jun. 2012.

[4] A. F. Brooks, T. L. Kelly, P. J. Veitch, and J. Munch, Ultra-sensitive wavefront measurement using a Hartmann sensor., Opt. Express, vol. 15, no. 16, pp. 10370-5, Aug. 2007.

[5] R. Day, Using the phase camera in advanced interferometers, GWADW 2013 (VIR-0310A-13)

[6] M. Lorenzini for the TCS team, AdV TCS, Virgo Week, January 2014, (VIR-0030A-14)

[7] K. Goda, D. Ottaway, B. Connelly, R. Adhikari, N. Mavalvala, and A. Gretarsson, Frequency-resolving spatiotemporal wave-front sensor., Opt. Lett., vol. 29, no. 13, pp. 1452-4, Jul. 2004.

[8] R. Day, E. Genin and A. Rocchi, Phase Camera requirements from INJ and TCS Subsystems, Virgo Technical Documentation System (VIR-0258A-12)

[9] Martin van Beuzekom, Jo van den Brand, and David S. Rabeling, ADC and DAQ Constraints for Digital Demodulation for the Advanced Virgo Phase Camera Virgo Technical Documentation System (VIR-0304A-12)

[10] D. Rabeling, K. Agatsuma, M. van Beuzekom, G. Visser, H. Verkooijen, W. Vink, and J. van den Brand, Phase camera status, Virgo Week, June 2013, (VIR-0281A-13) 